\title{
Market Returns to Education in Pakistan, Corrected for Endogeneity Bias
}

\author{
Sajjad Haider Bhatti*, Muhammad Aslam**and Jean \\ Bourdon $^{* * *}$
}

\begin{abstract}
This paper estimates the Mincer wage model for Pakistan's labor market, using a relatively recent dataset and new independent variables. We employ instrumental variables and two-stage least squares to address the problem of the endogeneity of education. Our results show that the returns to education are biased downward due to endogeneity, with significant wage gaps emerging among different regions, between genders and between urban and rural job markets. The study's choice of instruments has conceptual as well as empirical grounds. Our findings establish that the wage determination process is different for males and females across provincial labor markets.
\end{abstract}

Keywords: Endogeneity of education, human capital model, instrumental variables, Mincer regression, labor market, returns to schooling, Pakistan.

\section{JEL classification: C26.}

\section{Introduction}

The labor market remunerates workers based on their knowledge and the competencies they have acquired over their working life. Collectively, these skills comprise a worker's human capital. The interrelation between human capital and market earnings is captured by the human capital earnings function developed by Mincer (1958, 1974), Becker (1975) and Ben-Porath (1967). This model shows how formal education and on-the-job training (experience) are the key factors contributing to human capital. Mincer's (1974) model of human capital is commonly used to determine the impact of education and training on remuneration (see, for instance, Willis, 1986; Card, 1999). Even in its most basic form, the model

\footnotetext{
* Department of Statistics, Government College University, Faisalabad, Pakistan.

** Department of Statistics, Bahauddin Zakariya University, Multan, Pakistan.

*** IREDU- CNRS, Université de Bourgogne, Dijon, France.
} 
remains a key approach to calculating earning differentials associated with urban versus rural status, unionized versus non-unionized status, gender, ethnicity, public versus private sector work, and religion (see Johnson \& Chow, 1997; Kimmel, 1997; Lassibille, 1998; Korsun, 2010).

Despite the extensive application of the Mincer model, simple estimations using ordinary least squares (OLS) may be biased due to endogenous schooling variables and sample selectivity (Griliches, 1977, 1979; Angrist \& Krueger, 1991). The endogeneity problem arises when the explanatory variables are correlated with the error terms: this problem is built into the Mincer earnings function because all individuals in a wage market can never be exactly the same with respect to different unobservable and observable characteristics (Bhatti et al., 2013). Innate ability, for instance, is treated as a determinant of wages, but it is also correlated with schooling. Since innate ability is not directly measureable, simple OLS estimations are likely to yield biased results (Griliches, 1977; Card, 2001), with adverse implications for policymaking.

This endogeneity bias is generally addressed by estimating the human capital earnings function using an approach that combines instrumental variables with two-stage least squares (IV-2SLS), developed by Theil (1953) and Basmann (1957). The technique relies on different instruments of exogenous variation, including birth cohorts, the education level of other household members, IQ test scores, changes in education system and household sibling composition (Ashenfelter et al., 1999; Butcher \& Case, 1994). ${ }^{1}$ The literature suggests that the schooling coefficient is higher when one accounts for possible endogeneity using IV2SLS relative to simple OLS.

While many studies have estimated a human capital earnings function for Pakistan, most have not tackled econometric problems such as the potential endogeneity of the schooling variable. ${ }^{2}$ The few studies that do so (using instrumental variables) include Abbas and Foreman-Peck (2008), Aslam et al. (2008) and Aslam (2009). This points to ample room for quantifying the economic returns to education in the Pakistani labor market, corrected for endogeneity bias using new instruments. The rest of the article is structured as follows. Section 2 describes the data, variables and method used, including the nature of the instrumental variables involved. Section 3 presents the results of the Mincer model, estimated using OLS and IV-2SLS.

\footnotetext{
${ }^{1}$ For further reading, see the references given in Bhatti et al. (2013).

${ }^{2}$ See, among others, Qureshi (2012); Aslam and Kingdon (2009); Farooq and Sulaiman (2009); Hyder and Reilly (2005).
} 
The model is estimated separately for males and females and for the four provinces. Section 4 briefly discusses these results relative to the literature on Pakistan and Section 5 concludes the paper.

\section{Methodology}

We have used data from the Labor Force Survey conducted by the Pakistan Bureau of Statistics in 2008/09. After cleaning the data, we are left with 19,574 observations. We take the monthly wage of individuals as the response variable. The independent variables include different human capital and other social, economic and regional factors contributing to wage determination (Table 1).

\section{Table 1: Variables used to estimate Mincer earnings function}

\begin{tabular}{|c|c|}
\hline Variable & Definition \\
\hline \multicolumn{2}{|c|}{ Dependent variable } \\
\hline Lnwage & Natural logarithm of the individual's monthly wage (main job) \\
\hline \multicolumn{2}{|c|}{ Independent variables } \\
\hline Education & Number of years of schooling completed \\
\hline Experience & Potential experience measured in years (age $-6-\mathrm{SCH} 1$ ) \\
\hline Experience2 & Potential experience squared \\
\hline Hours & $\begin{array}{l}\text { Number of hours accounting for monthly salary (hours worked } \\
\text { per month) }\end{array}$ \\
\hline Gender & Gender $($ male $=1$, female $=0)$ \\
\hline Urban & $\begin{array}{l}\text { Dummy variable indicating where the individual's work is } \\
\text { located (urban }=1 \text {, rural }=0 \text { ) }\end{array}$ \\
\hline Professional & $\begin{array}{l}\text { Dummy variable indicating whether the individual has a } \\
\text { professional or general degree/diploma (professional = } 1 \text {, general } \\
=0 \text { ) }\end{array}$ \\
\hline Public & $\begin{array}{l}\text { Dummy variable indicating whether the individual works in the } \\
\text { public or private sector (public }=1 \text {, private }=0 \text { ) }\end{array}$ \\
\hline Tmp.contract & $\begin{array}{l}\text { Dummy variable indicating that the individual has temporary } \\
\text { employment (without contract) (reference category) }\end{array}$ \\
\hline Fix.contract & $\begin{array}{l}\text { Dummy variable indicating whether the individual has a fixed- } \\
\text { term contract (yes }=1 \text {, otherwise } 0 \text { ) }\end{array}$ \\
\hline Perm.contract & $\begin{array}{l}\text { Dummy variable indicating whether the individual has a } \\
\text { permanent contract (yes }=1 \text {, otherwise } 0 \text { ) }\end{array}$ \\
\hline Punjab & $\begin{array}{l}\text { Dummy variable indicating that the individual is from Punjab } \\
\text { (reference category) }\end{array}$ \\
\hline Sindh & $\begin{array}{l}\text { Dummy variable indicating whether the individual is from Sindh } \\
\text { (yes }=1 \text {, otherwise } 0 \text { ) }\end{array}$ \\
\hline $\mathrm{KP}$ & $\begin{array}{l}\text { Dummy variable indicating whether the individual is from } \mathrm{KP} \\
\text { (yes }=1 \text {, otherwise } 0 \text { ) }\end{array}$ \\
\hline Balochistan & $\begin{array}{l}\text { Dummy variable indicating whether the individual is from } \\
\text { Balochistan (yes }=1 \text {, otherwise } 0 \text { ) }\end{array}$ \\
\hline
\end{tabular}


Identifying a relevant valid instrument is difficult (Bound et al., 1995). While many studies have used different instruments in IV-2SLS estimations of the earnings function, the issue of endogeneity bias persists (Angrist \& Krueger, 1992). We estimate the Mincer earnings function for Pakistani wage market data, using two instrumental variables.

- Instrument 1 is the average numbers of years of schooling attained in the enumeration block where the individual's household is located. The rationale for using this instrument is to combine the effects of several commonly used exogenous factors, including distance to school, general trend toward education, and social environment.

- Instrument 2 follows a similar logic, but also accounts for other factors at the time the individual leaves formal schooling and joins the workforce. Following Bhatti et al. (2013), we define Instrument 2 as the average number of years of schooling attained by a given gender and age group in the year the individual joined the labor market. The data is taken from Barro and Lee (2010), who have estimated the average number of years of schooling for a large sample of countries for 1950-2010. ${ }^{3}$

\section{Data Analysis and Results}

Tables $2-5$ give the results of the estimated human capital earnings function for the Pakistani labor market. The first column in each case presents the OLS results (uncorrected for potential endogeneity bias), while the remaining columns present the IV-2SLS estimation results using both instrumental variables. In the first-stage regressions, the substantially low pvalues (given in parentheses) linked to the instrumental variables indicate that both can be considered relevant instruments. The validity of these instruments is assessed using the Sargan test, which indicates that they are valid for Balochistan, but only for females in the other three provinces.

The analysis employs separate Mincer wage models for males and females in all four provinces. Tables 2-5 give the OLS and IV-2SLS estimation results for males and females in Punjab, Sindh, Khyber Pakhtunkhwa (KP) and Balochistan, respectively. In all four cases, the Hausman (1978) exogeneity test statistic indicates significant bias due to the endogeneity of the schooling variable. A comparison of the OLS and IV-2SLS results for endogenous schooling reveals a downward bias associated with this endogeneity. The downward bias in returns to

\footnotetext{
${ }^{3}$ These measures of average schooling by gender and age group for different countries are available at www.barrolee.com.
} 
schooling is higher for males in Sindh and KP, and for females in Punjab, but similar for both in Balochistan.

The higher coefficient in the IV-2SLS estimations is in line with international studies such as Butcher and Case (1994), Card (1999), Ashenfelter et al. (1999) and Chen and Hamori (2009) as well as Pakistanbased studies such as Abbas and Foreman-Peck (2008) and Aslam (2009). Bhatti et al. (2013) find similar differences in endogeneity-corrected and uncorrected returns to schooling, using similar instruments applied to data for France.

Table 2: OLS and IV-2SLS estimation results, Punjab

\begin{tabular}{|c|c|c|c|c|c|c|}
\hline \multirow[b]{2}{*}{ Variable } & \multicolumn{3}{|c|}{ Males } & \multicolumn{3}{|c|}{ Females } \\
\hline & OLS & $\begin{array}{l}\text { First } \\
\text { stage }\end{array}$ & $\begin{array}{l}\text { Second } \\
\text { stage }\end{array}$ & OLS & $\begin{array}{l}\text { First } \\
\text { stage }\end{array}$ & $\begin{array}{l}\text { Second } \\
\text { stage }\end{array}$ \\
\hline Intercept & $\begin{array}{c}8.0639 \\
(<0.0001)\end{array}$ & $\begin{array}{c}-5.2761 \\
(<0.0001)\end{array}$ & $\begin{array}{c}7.7622 \\
(<0.0001)\end{array}$ & $\begin{array}{c}8.3405 \\
(<0.0001)\end{array}$ & $\begin{array}{c}30.0627 \\
(<0.0001)\end{array}$ & $\begin{array}{c}7.6287 \\
(<0.0001)\end{array}$ \\
\hline Education & $\begin{array}{c}0.0507 \\
(<0.0001)\end{array}$ & - & $\begin{array}{c}0.0755 \\
(<0.0001)\end{array}$ & $\begin{array}{c}0.0504 \\
(<0.0001)\end{array}$ & - & $\begin{array}{c}0.085 \\
(<0.0001)\end{array}$ \\
\hline Exper & $\begin{array}{c}0.0308 \\
(<0.0001)\end{array}$ & $\begin{array}{c}0.0235 \\
(<0.0001)\end{array}$ & $\begin{array}{c}0.0366 \\
(<0.0001)\end{array}$ & $\begin{array}{c}0.0299 \\
(<0.0001)\end{array}$ & $\begin{array}{c}-0.7469 \\
(<0.0001)\end{array}$ & $\begin{array}{c}0.0381 \\
(<0.0001)\end{array}$ \\
\hline Experience $^{2}$ & $\begin{array}{c}-0.0004 \\
(<0.0001)\end{array}$ & $\begin{array}{c}0.0002 \\
(<0.0001)\end{array}$ & $\begin{array}{c}-0.0004 \\
(<0.0001)\end{array}$ & $\begin{array}{l}-0.0005 \\
(0.0046)\end{array}$ & $\begin{array}{c}0.0054 \\
(<0.0001)\end{array}$ & $\begin{array}{l}-0.0004 \\
(0.0104)\end{array}$ \\
\hline Hours & $\begin{array}{c}0.0011 \\
(0.2055)\end{array}$ & $\begin{array}{l}-0.0183 \\
(0.0028)\end{array}$ & $\begin{array}{c}0.0018 \\
(0.05023)\end{array}$ & $\begin{array}{l}-0.0015 \\
(0.6248)\end{array}$ & $\begin{array}{c}-0.1073 \\
(<0.0001)\end{array}$ & $\begin{array}{c}0.0039 \\
(0.2630)\end{array}$ \\
\hline Urban & $\begin{array}{c}0.1115 \\
(0.0016)\end{array}$ & $\begin{array}{l}-0.1426 \\
(0.5558)\end{array}$ & $\begin{array}{c}0.1089 \\
(0.0025)\end{array}$ & $\begin{array}{c}-0.0129 \\
(0.9042)\end{array}$ & $\begin{array}{l}-0.0084 \\
(0.9898)\end{array}$ & $\begin{array}{l}-0.0194 \\
(0.8614)\end{array}$ \\
\hline Professional & $\begin{array}{c}0.5445 \\
(<0.0001)\end{array}$ & $\begin{array}{c}2.8599 \\
(<0.0001)\end{array}$ & $\begin{array}{c}0.4038 \\
(<0.0001)\end{array}$ & $\begin{array}{c}0.8179 \\
(<0.0001)\end{array}$ & $\begin{array}{c}2.7708 \\
(0.0009)\end{array}$ & $\begin{array}{c}0.6765 \\
(<0.0001)\end{array}$ \\
\hline Public & $\begin{array}{c}-0.0912 \\
(<0.0001)\end{array}$ & $\begin{array}{c}-0.6382 \\
(<0.0001)\end{array}$ & $\begin{array}{c}-0.0638 \\
(<0.0001)\end{array}$ & $\begin{array}{c}-0.1849 \\
(<0.0001)\end{array}$ & $\begin{array}{l}-1.0436 \\
(<0.0001)\end{array}$ & $\begin{array}{l}-0.1368 \\
(0.0024)\end{array}$ \\
\hline Fix.contract & $\begin{array}{c}0.0454 \\
(0.1341)\end{array}$ & $\begin{array}{c}1.4152 \\
(<0.0001)\end{array}$ & $\begin{array}{l}-0.0103 \\
(0.7451)\end{array}$ & $\begin{array}{l}-0.0065 \\
(0.9375)\end{array}$ & $\begin{array}{c}1.3017 \\
(0.0103)\end{array}$ & $\begin{array}{l}-0.0775 \\
(0.3786)\end{array}$ \\
\hline Perm & $\begin{array}{c}0.2822 \\
(<0.0001)\end{array}$ & $\begin{array}{c}2.7877 \\
(<0.0001)\end{array}$ & $\begin{array}{c}0.1709 \\
(<0.0001)\end{array}$ & $\begin{array}{c}0.3006 \\
(<0.0001)\end{array}$ & $\begin{array}{c}2.5786 \\
(<0.0001)\end{array}$ & $\begin{array}{c}0.1553 \\
(0.0497)\end{array}$ \\
\hline Instrument 1 & - & $\begin{array}{c}0.6752 \\
(<0.0001)\end{array}$ & - & - & $\begin{array}{c}0.5424 \\
(<0.0001)\end{array}$ & - \\
\hline Instrument 2 & - & $\begin{array}{c}1.6564 \\
(<0.0001)\end{array}$ & - & - & $\begin{array}{c}-3.2193 \\
(<0.0001)\end{array}$ & - \\
\hline $\begin{array}{l}\text { Hausman exogeneity } \\
\text { test }\end{array}$ & - & $\begin{array}{c}72.34 \\
(<0.0001)\end{array}$ & & - & $\begin{array}{c}15.38 \\
(<0.0001)\end{array}$ & \\
\hline Sargan-Hansen test & - & $\begin{array}{c}24.77 \\
(0.0002)\end{array}$ & & - & $\begin{array}{c}0.01 \\
(0.9208)\end{array}$ & \\
\hline $\mathrm{R}^{2}$ & 0.4087 & 0.5836 & 0.384 & 0.4417 & 0.7104 & 0.4045 \\
\hline F/Wald statistic & 290.9 & 530.6 & 257.1 & 39.9 & 111.1 & 37 \\
\hline
\end{tabular}

Source: Authors' estimates. 
Table 2 presents the OLS and IV-2SLS estimation results for males and females working in Punjab. The returns to schooling are 7.55 percent for males and 8.05 percent for females, implying that every additional year spent in school increases wages by 7.76 percent. The returns to labor market experience are slightly higher in the IV-2SLS estimation: each additional year of labor market experience increases monthly wages by 3.6 percent for males and 3.8 percent for females. The negative coefficient of work experience squared indicates that the returns to experience are concave, that is, the rate of increase tends to fall as labor market experience rises.

The coefficient of work location (urban or rural) is significant only for males. Urban male workers in Punjab earn nearly 10.89 percent more than their rural counterparts. Male workers with a professional degree earn 40 percent more than those with a general qualification. The corresponding wage premium for females is 67 percent. The wage loss associated with working in the public sector is about 6.3 percent for males and about 13 percent for females. Finally, the results show that males with permanent employment earn about 17.09 percent more than those on a fixed-term contract. The corresponding wage premium for females is 15.53 percent. The subsequent tables (Table 3-5) reveal similar results. 
Table 3: OLS and IV-2SLS estimation results, Sindh

\begin{tabular}{|c|c|c|c|c|c|c|}
\hline \multirow[b]{2}{*}{ Variable } & \multicolumn{3}{|c|}{ Males } & \multicolumn{3}{|c|}{ Females } \\
\hline & OLS & $\begin{array}{l}\text { First } \\
\text { stage }\end{array}$ & $\begin{array}{l}\text { Second } \\
\text { stage }\end{array}$ & OLS & $\begin{array}{l}\text { First } \\
\text { stage }\end{array}$ & $\begin{array}{l}\text { Second } \\
\text { stage }\end{array}$ \\
\hline Intercept & $\begin{array}{c}7.8893 \\
(<0.0001)\end{array}$ & $\begin{array}{l}-4.9993 \\
(<0.0001)\end{array}$ & $\begin{array}{c}7.2441 \\
(<0.0001)\end{array}$ & $\begin{array}{c}8.0007 \\
(<0.0001)\end{array}$ & $\begin{array}{l}31.4907 \\
(<0.0001)\end{array}$ & $\begin{array}{c}7.2394 \\
(<0.0001)\end{array}$ \\
\hline Education & $\begin{array}{c}0.0636 \\
(<0.0001)\end{array}$ & - & $\begin{array}{c}0.1136 \\
(<0.0001)\end{array}$ & $\begin{array}{c}0.0624 \\
(<0.0001)\end{array}$ & - & $\begin{array}{c}0.0981 \\
(<0.0001)\end{array}$ \\
\hline Experience & $\begin{array}{c}0.0363 \\
(<0.0001)\end{array}$ & $\begin{array}{c}0.1119 \\
(<0.0001)\end{array}$ & $\begin{array}{c}0.0425 \\
(<0.0001)\end{array}$ & $\begin{array}{c}0.0099 \\
(0.3424)\end{array}$ & $\begin{array}{l}-0.7253 \\
(0.0019)\end{array}$ & $\begin{array}{c}0.0159 \\
(0.0148)\end{array}$ \\
\hline Experience $^{2}$ & $\begin{array}{c}-0.0004 \\
(<0.0001)\end{array}$ & $\begin{array}{c}-0.0018 \\
(<0.0001)\end{array}$ & $\begin{array}{c}-0.0004 \\
(<0.0001)\end{array}$ & $\begin{array}{c}-0.0089 \\
(0.9238)\end{array}$ & $\begin{array}{c}0.0058 \\
(0.0019)\end{array}$ & $\begin{array}{c}-0.0086 \\
(0.9182)\end{array}$ \\
\hline Hours & $\begin{array}{c}0.003 \\
(0.0031)\end{array}$ & $\begin{array}{c}-0.0363 \\
(<0.0001)\end{array}$ & $\begin{array}{c}0.0048 \\
(<0.0001)\end{array}$ & $\begin{array}{c}0.012 \\
(0.0051)\end{array}$ & $\begin{array}{c}-0.1457 \\
(<0.0001)\end{array}$ & $\begin{array}{c}0.019 \\
(0.0002)\end{array}$ \\
\hline Urban & $\begin{array}{l}-0.0039 \\
(0.9283)\end{array}$ & $\begin{array}{l}-0.4386 \\
(0.1440)\end{array}$ & $\begin{array}{c}0.0253 \\
(0.5894)\end{array}$ & $\begin{array}{l}-0.3076 \\
(0.1774)\end{array}$ & $\begin{array}{l}-0.2443 \\
(0.8565)\end{array}$ & $\begin{array}{l}-0.3351 \\
(0.1556)\end{array}$ \\
\hline Professional & $\begin{array}{c}0.2709 \\
(<0.0001)\end{array}$ & $\begin{array}{c}2.372 \\
(0.0001)\end{array}$ & $\begin{array}{c}0.0299 \\
(<0.0001)\end{array}$ & $\begin{array}{c}0.6767 \\
(<0.0001)\end{array}$ & $\begin{array}{c}2.6867 \\
(0.0005)\end{array}$ & $\begin{array}{c}0.5365 \\
(0.0003)\end{array}$ \\
\hline Public & $\begin{array}{l}-0.1057 \\
(<0.0001)\end{array}$ & $\begin{array}{l}-0.6558 \\
(<0.0001)\end{array}$ & $\begin{array}{l}-0.0523 \\
(<0.0001)\end{array}$ & $\begin{array}{l}-0.1052 \\
(0.0610)\end{array}$ & $\begin{array}{l}-1.0217 \\
(0.0016)\end{array}$ & $\begin{array}{l}-0.0324 \\
(0.6061)\end{array}$ \\
\hline Fix.contract & $\begin{array}{c}0.0572 \\
(0.0828)\end{array}$ & $\begin{array}{c}0.9634 \\
(0.0001)\end{array}$ & $\begin{array}{l}-0.0344 \\
(0.3433)\end{array}$ & $\begin{array}{c}-0.14 \\
(0.3001)\end{array}$ & $\begin{array}{c}0.2583 \\
(0.7461)\end{array}$ & $\begin{array}{c}-0.163 \\
(0.2437)\end{array}$ \\
\hline Perm.contract & $\begin{array}{c}0.2782 \\
(<0.0001)\end{array}$ & $\begin{array}{c}2.333 \\
(<0.0001)\end{array}$ & $\begin{array}{c}0.0511 \\
(0.07691)\end{array}$ & $\begin{array}{c}0.2883 \\
(0.0011)\end{array}$ & $\begin{array}{c}1.5566 \\
(0.0026)\end{array}$ & $\begin{array}{c}0.1757 \\
(0.0747)\end{array}$ \\
\hline Instrument 1 & - & $\begin{array}{c}0.6687 \\
(<0.0001)\end{array}$ & - & - & $\begin{array}{c}0.5857 \\
(<0.0001)\end{array}$ & - \\
\hline Instrument 2 & - & $\begin{array}{c}1.7457 \\
(<0.0001)\end{array}$ & - & - & $\begin{array}{l}-3.3918 \\
(<0.0001)\end{array}$ & - \\
\hline $\begin{array}{l}\text { Hausman exogeneity } \\
\text { test }\end{array}$ & - & $\begin{array}{l}307.450 \\
(<0.0001)\end{array}$ & & - & $\begin{array}{c}19.620 \\
(<0.0001)\end{array}$ & \\
\hline Sargan-Hansen test & - & $\begin{array}{c}4.926 \\
(0.0265)\end{array}$ & & - & $\begin{array}{c}1.461 \\
(0.2268)\end{array}$ & \\
\hline $\mathrm{R}^{2}$ & 0.5085 & 0.6068 & 0.4210 & 0.4809 & 0.6842 & 0.4453 \\
\hline F/Wald statistic & 387.4 & 519.9 & 330.7 & 25.63 & 53.73 & 23.29 \\
\hline
\end{tabular}

Source: Authors' estimates. 
Table 4: OLS and IV-2SLS estimation results, KP

\begin{tabular}{|c|c|c|c|c|c|c|}
\hline \multirow[b]{2}{*}{ Variable } & \multicolumn{3}{|c|}{ Males } & \multicolumn{3}{|c|}{ Females } \\
\hline & OLS & $\begin{array}{l}\text { First } \\
\text { stage }\end{array}$ & $\begin{array}{c}\text { Second } \\
\text { stage }\end{array}$ & OLS & $\begin{array}{l}\text { First } \\
\text { stage }\end{array}$ & $\begin{array}{c}\text { Second } \\
\text { stage }\end{array}$ \\
\hline Intercept & $\begin{array}{c}7.8986 \\
(<0.0001)\end{array}$ & $\begin{array}{c}-4.603 \\
(0.0348)\end{array}$ & $\begin{array}{c}7.5054 \\
(<0.0001)\end{array}$ & $\begin{array}{c}7.5098 \\
(<0.0001)\end{array}$ & $\begin{array}{c}33.5428 \\
(<0.0001)\end{array}$ & $\begin{array}{c}7.2194 \\
(<0.0001)\end{array}$ \\
\hline Education & $\begin{array}{c}0.0559 \\
(<0.0001)\end{array}$ & - & $\begin{array}{c}0.0804 \\
(<0.0001)\end{array}$ & $\begin{array}{c}0.0631 \\
(<0.0001)\end{array}$ & - & $\begin{array}{c}0.0756 \\
(0.0014)\end{array}$ \\
\hline Experience & $\begin{array}{c}0.0389 \\
(<0.0001)\end{array}$ & $\begin{array}{c}0.1598 \\
(0.0010)\end{array}$ & $\begin{array}{c}0.0421 \\
(<0.0001)\end{array}$ & $\begin{array}{c}0.0245 \\
(0.0564)\end{array}$ & $\begin{array}{l}-0.6386 \\
(0.0001)\end{array}$ & $\begin{array}{c}0.0258 \\
(0.0486)\end{array}$ \\
\hline Experience $^{2}$ & $\begin{array}{c}-0.0004 \\
(<0.0001)\end{array}$ & $\begin{array}{l}-0.0027 \\
(0.0001)\end{array}$ & $\begin{array}{c}-0.0004 \\
(<0.0001)\end{array}$ & $\begin{array}{l}-0.0012 \\
(0.887)\end{array}$ & $\begin{array}{c}0.0028 \\
(0.2571)\end{array}$ & $\begin{array}{c}0.0001 \\
(0.7846)\end{array}$ \\
\hline Hours & $\begin{array}{l}0.0002 \\
(0.976)\end{array}$ & $\begin{array}{c}-0.0734 \\
(<0.0001)\end{array}$ & $\begin{array}{c}0.0025 \\
(0.1261)\end{array}$ & $\begin{array}{c}0.012 \\
(0.0329)\end{array}$ & $\begin{array}{c}-0.1979 \\
(<0.0001)\end{array}$ & $\begin{array}{c}0.0151 \\
(0.0406)\end{array}$ \\
\hline Urban & $\begin{array}{c}0.0718 \\
(0.1797)\end{array}$ & $\begin{array}{l}-0.3079 \\
(0.4642)\end{array}$ & $\begin{array}{c}0.0672 \\
(0.2202)\end{array}$ & $\begin{array}{l}-0.0155 \\
(0.9175)\end{array}$ & $\begin{array}{c}2.597 \\
(0.0103)\end{array}$ & $\begin{array}{l}-0.0471 \\
(0.7662)\end{array}$ \\
\hline Professional & $\begin{array}{c}0.5952 \\
(<0.0001)\end{array}$ & $\begin{array}{c}2.5934 \\
(<0.0001)\end{array}$ & $\begin{array}{c}0.481 \\
(<0.0001)\end{array}$ & $\begin{array}{c}0.3895 \\
(0.3004)\end{array}$ & $\begin{array}{c}0.8641 \\
(0.73911)\end{array}$ & $\begin{array}{c}0.3569 \\
(0.3496)\end{array}$ \\
\hline Public & $\begin{array}{l}-0.0351 \\
(0.1518)\end{array}$ & $\begin{array}{c}-1.1535 \\
(<0.0001)\end{array}$ & $\begin{array}{c}0.0064 \\
(0.8127)\end{array}$ & $\begin{array}{l}-0.3604 \\
(0.0001)\end{array}$ & $\begin{array}{l}-1.2231 \\
(0.0411)\end{array}$ & $\begin{array}{c}-0.3423 \\
(0.0003)\end{array}$ \\
\hline Fix.contract & $\begin{array}{c}0.2128 \\
(0.0003)\end{array}$ & $\begin{array}{c}1.0571 \\
(0.0206)\end{array}$ & $\begin{array}{c}0.1703 \\
(0.0049)\end{array}$ & $\begin{array}{c}0.2256 \\
(0.2325)\end{array}$ & $\begin{array}{l}-0.5132 \\
(0.6192)\end{array}$ & $\begin{array}{c}0.226 \\
(0.2339)\end{array}$ \\
\hline Perm.contract & $\begin{array}{c}0.1111 \\
(0.0018)\end{array}$ & $\begin{array}{c}2.12 \\
(<0.0001)\end{array}$ & $\begin{array}{c}0.0294 \\
(0.4825)\end{array}$ & $\begin{array}{c}0.3589 \\
(0.0042)\end{array}$ & $\begin{array}{l}-0.4048 \\
(0.0063)\end{array}$ & $\begin{array}{c}0.3502 \\
(0.0057)\end{array}$ \\
\hline Instrument 1 & - & $\begin{array}{c}0.6147 \\
(<0.0001)\end{array}$ & - & - & $\begin{array}{c}0.4174 \\
(<0.0001)\end{array}$ & - \\
\hline Instrument 2 & - & $\begin{array}{c}2.0966 \\
(<0.0001) \\
\end{array}$ & - & - & $\begin{array}{l}-2.9922 \\
(0.0003) \\
\end{array}$ & - \\
\hline $\begin{array}{l}\text { Hausman exogeneity } \\
\text { test }\end{array}$ & - & $\begin{array}{c}16.446 \\
(<0.0001)\end{array}$ & & - & $\begin{array}{c}18.446 \\
(<0.0001)\end{array}$ & \\
\hline Sargan-Hansen test & - & $\begin{array}{c}6.964 \\
(0.0083)\end{array}$ & & - & $\begin{array}{c}0.558 \\
(0.4550)\end{array}$ & \\
\hline $\mathrm{R}^{2}$ & 0.4308 & 0.5666 & 0.4044 & 0.4521 & 0.5967 & 0.4470 \\
\hline F/Wald statistic & 113.3 & 175.9 & 88.45 & 13.57 & 21.75 & 10.81 \\
\hline
\end{tabular}

Source: Authors' estimates. 
Table 5: OLS and IV-2SLS estimation results, Balochistan

\begin{tabular}{|c|c|c|c|c|c|c|}
\hline \multirow[b]{2}{*}{ Variable } & \multicolumn{3}{|c|}{ Males } & \multicolumn{3}{|c|}{ Females } \\
\hline & OLS & $\begin{array}{l}\text { First } \\
\text { stage }\end{array}$ & $\begin{array}{c}\text { Second } \\
\text { stage }\end{array}$ & OLS & $\begin{array}{l}\text { First } \\
\text { stage }\end{array}$ & $\begin{array}{c}\text { Second } \\
\text { stage }\end{array}$ \\
\hline Intercept & $\begin{array}{c}8.5959 \\
(<0.0001)\end{array}$ & $\begin{array}{c}-8.7668 \\
(<0.0001)\end{array}$ & $\begin{array}{c}8.3309 \\
(<0.0001)\end{array}$ & $\begin{array}{c}8.1682 \\
(<0.0001)\end{array}$ & $\begin{array}{c}32.515 \\
(0.0001)\end{array}$ & $\begin{array}{c}7.8487 \\
(<0.0001)\end{array}$ \\
\hline Education & $\begin{array}{c}0.0533 \\
(<0.0001)\end{array}$ & - & $\begin{array}{c}0.0702 \\
(<0.0001)\end{array}$ & $\begin{array}{c}0.0594 \\
(<0.0001)\end{array}$ & - & $\begin{array}{c}0.0752 \\
(<0.0001)\end{array}$ \\
\hline Experience & $\begin{array}{c}0.028 \\
(<0.0001)\end{array}$ & $\begin{array}{c}0.2843 \\
(<0.0001)\end{array}$ & $\begin{array}{c}0.0292 \\
(<0.0001)\end{array}$ & $\begin{array}{c}0.0365 \\
(0.0095)\end{array}$ & $\begin{array}{c}-0.9402 \\
(<0.0001)\end{array}$ & $\begin{array}{c}0.0416 \\
(0.0051)\end{array}$ \\
\hline Experience $^{2}$ & $\begin{array}{l}-0.0003 \\
(0.0019)\end{array}$ & $\begin{array}{c}-0.0044 \\
(<0.0001)\end{array}$ & $\begin{array}{l}-0.0002 \\
(0.0107)\end{array}$ & $\begin{array}{l}-0.0005 \\
(0.1438)\end{array}$ & $\begin{array}{c}0.0063 \\
(0.1139)\end{array}$ & $\begin{array}{l}-0.0006 \\
(0.1315)\end{array}$ \\
\hline Hours & $\begin{array}{l}-0.0041 \\
(0.0321)\end{array}$ & $\begin{array}{c}-0.0685 \\
(<0.0001)\end{array}$ & $\begin{array}{l}-0.0024 \\
(0.2383)\end{array}$ & $\begin{array}{c}-0.004 \\
(0.4762)\end{array}$ & $\begin{array}{l}-0.1585 \\
(0.0064)\end{array}$ & $\begin{array}{c}0.0002 \\
(0.9743)\end{array}$ \\
\hline Urban & $\begin{array}{l}-0.0766 \\
(0.1466)\end{array}$ & $\begin{array}{c}0.5578 \\
(0.1605)\end{array}$ & $\begin{array}{c}-0.091 \\
(0.0897)\end{array}$ & $\begin{array}{l}-0.1714 \\
(0.6141)\end{array}$ & $\begin{array}{c}0.4092 \\
(0.9061)\end{array}$ & $\begin{array}{l}-0.1728 \\
(0.6186)\end{array}$ \\
\hline Professional & $\begin{array}{c}0.2428 \\
(0.0022)\end{array}$ & $\begin{array}{c}2.8487 \\
(<0.0001)\end{array}$ & $\begin{array}{c}0.1564 \\
(0.0693)\end{array}$ & $\begin{array}{c}0.1079 \\
(0.5476)\end{array}$ & $\begin{array}{c}3.0128 \\
(0.0931)\end{array}$ & $\begin{array}{l}0.0402 \\
(0.831)\end{array}$ \\
\hline Public & $\begin{array}{c}-0.1226 \\
(<0.0001)\end{array}$ & $\begin{array}{c}-1.3242 \\
(<0.0001)\end{array}$ & $\begin{array}{c}-0.086 \\
(0.0026)\end{array}$ & $\begin{array}{l}-0.0281 \\
(0.8468)\end{array}$ & $\begin{array}{c}2.0174 \\
(0.1734)\end{array}$ & $\begin{array}{c}-0.0422 \\
(<0.0001)\end{array}$ \\
\hline Fix.contract & $\begin{array}{l}-0.0879 \\
(0.0396)\end{array}$ & $\begin{array}{c}-0.103 \\
(0.7515)\end{array}$ & $\begin{array}{l}-0.1025 \\
(0.0184)\end{array}$ & $\begin{array}{l}-0.2649 \\
(0.1292)\end{array}$ & $\begin{array}{c}0.4209 \\
(0.8129)\end{array}$ & $\begin{array}{c}-0.2753 \\
(0.123)\end{array}$ \\
\hline Perm.contract & $\begin{array}{c}0.1042 \\
(0.0034)\end{array}$ & $\begin{array}{c}1.9 \\
(<0.0001)\end{array}$ & $\begin{array}{c}0.0551 \\
(0.1686)\end{array}$ & $\begin{array}{c}0.3771 \\
(0.0063)\end{array}$ & $\begin{array}{c}2.6163 \\
(0.0514)\end{array}$ & $\begin{array}{c}0.3077 \\
(0.0363)\end{array}$ \\
\hline Instrument 1 & - & $\begin{array}{c}0.5425 \\
(<0.0001)\end{array}$ & - & - & $\begin{array}{c}0.6752 \\
(0.0001)\end{array}$ & - \\
\hline Instrument 2 & - & $\begin{array}{c}2.6031 \\
(<0.0001)\end{array}$ & - & - & $\begin{array}{l}-4.5426 \\
(0.0001)\end{array}$ & - \\
\hline $\begin{array}{l}\text { Hausman exogeneity } \\
\text { test }\end{array}$ & - & $\begin{array}{c}7.800 \\
(0.0053)\end{array}$ & & - & $\begin{array}{c}17.533 \\
(<0.0001)\end{array}$ & \\
\hline Sargan-Hansen test & - & $\begin{array}{c}2.469 \\
(0.1161)\end{array}$ & & - & $\begin{array}{c}0.692 \\
(0.4050)\end{array}$ & \\
\hline $\mathrm{R}^{2}$ & 0.4101 & 0.6053 & 0.3976 & 0.6348 & 0.6897 & 0.6189 \\
\hline F/Wald statistic & 100.4 & 199.3 & 80.39 & 14.87 & 16.89 & 12.65 \\
\hline
\end{tabular}

Source: Authors' estimates.

Following Bhatti et al. (2013), we attempt to choose the most appropriate instrumental variable of the two we have proposed through a correlation analysis. Tables A1 to A8 (in the Appendix) provide partial correlation matrices (with p-values in parentheses) of the response variable, suspected endogenous variable (schooling) and both instruments. We observe that, in most cases, instrument 2 has a lower correlation with endogenous schooling and a higher correlation with the response variable, 
which negates its value as a good instrument. On the other hand, instrument 1 has a reasonably strong correlation with the endogenous variable and a smaller correlation with the response variable, which implies it is a more relevant and appropriate instrumental variable. Keeping these correlation measures in view and its conceptual superiority, we give preference to instrument 1 as being more appropriate to the IV-2SLS estimation. Another advantage is that it is generated from the data itself.

\section{Discussion}

Since the Hausman (1978) test statistic indicates that schooling is endogenous and we consider the IV-2SLS estimation technique more suitable than OLS, this section discusses the IV-2SLS results in more detail. These results show that returns to schooling are, in most cases, 50 percent higher than the OLS results would indicate, which is in line with the results obtained by Bhatti et al. (2013), Butcher and Case (1994) and Card (1993). Returns to labor market experience are slightly higher in the IV-2SLS estimation, with each additional year of labor market experience causing monthly wages to rise at a decreasing rate.

As with Barnet-Verzat and Wolff (2008) and Araï et al. (1996), we find significant gender wage differentials in the returns to education. This is also in line with other Pakistan-based studies such as Yasin et al. (2010), Hyder (2007) and Khan and Toor (2003). The coefficient of work location (urban or rural) indicates that urban males earn more than their rural counterparts, except in Balochistan. The reverse holds true for female workers. A significant wage gap associated with location emerges for Punjab alone. This finding is similar in direction but smaller in magnitude to that of Farooq and Sulaiman (2009).

Our results show that, when corrected for the endogeneity of schooling, the wage premium of holding a professional degree falls substantially for both genders in all provinces. The IV-2SLS estimation indicates that people with a professional degree earn more than those without, reflecting the results obtained by Hyder (2007) and Khan and Toor (2003). However, this difference is not statistically significant for females in $\mathrm{KP}$ and Balochistan. Generally, there is a significant wage loss associated with working in the public sector compared to the private sector for both male and female workers. This finding contradicts Hyder (2007), who reports higher earnings for public sector workers in Pakistan. 
We also find that people with permanent employment earn more than those on a fixed-term or temporary contract, with male workers in KP being the exception. Our findings concerning provincial effects are in line with many other studies on the Pakistani labor market, including Farooq and Sulaiman (2009) and Khan and Toor (2003). A seeming discrepancy is that, generally, both specifications yield an $\mathrm{R}^{2}$ term of near 40 percent, which looks small but is common in cross-sectional studies (see Gujarati, 2007).

\section{Conclusion}

This study has used two new instrumental variables to correct for the possibility of endogeneity bias in schooling. The results reveal the significance of this endogeneity bias and the relevance of both instrumental variables. We find that instrument 1 - the average number of years of education in the enumeration block where the individual's household is located - is superior on statistical as well as conceptual grounds.

The results show that each additional year of schooling increases wages by 7.5 percent (for males), 8.5 percent (for females), 11.3 percent (for males), 9.8 percent (for females), 8.4 percent (for males), 7.6 percent (for females), 7.0 percent (for males) and 7.5 percent (for females) in Punjab, Sindh, KP and Balochistan, respectively. Urban wage markets offer some benefits, but not as much in terms of magnitude and significance. There is a significant wage gain for those with a professional degree. Finally, permanent employment is better remunerated than fixed-contract work. 


\section{References}

Abbas, Q., \& Foreman-Peck, J. (2008). The Mincer human capital model in Pakistan: Implications for education policy. South Asia Economic Journal, 9(2), 435-462.

Angrist, J. D., \& Krueger, A. B. (1991). Does compulsory school attendance affect schooling and earnings? Quarterly Journal of Economics, 106(4), 979-1014.

Angrist, J. D., \& Krueger, A. B. (1992). Estimating the payoff to schooling using the Vietnam-era draft lottery (Working Paper No. 4067). Cambridge, MA: National Bureau of Economic Research.

Araï, M., Ballot, G., \& Skalli, A. (1996). Différentiels intersectoriels de salaire et caractéristiques des employeurs en France. Economie et statistique, 299, 37-58.

Ashenfelter, O., Harmon, C., \& Oosterbeek, H. (1999). A review of estimates of the schooling/earnings relationship, with tests for publication bias. Labour Economics, 6(4), 453-470.

Aslam, M. (2009). Education gender gaps in Pakistan: Is the labor market to blame? Economic Development and Cultural Change, 57(4), 747-784.

Aslam, M., \& Kingdon, G. (2009). Public-private sector segmentation in the Pakistani labor market. Journal of Asian Economics, 20(1), 34-49.

Aslam, M., Bari, F., \& Kingdon, G. (2012). Returns to schooling, ability and cognitive skills in Pakistan. Education Economics, 20(2), 139-173.

Barnet-Verzat, C., \& Wolff, F.-C. (2008). Gender wage gap and the glass ceiling effect: A firm-level investigation. International Journal of Manpower, 29(6), 486-502.

Barro, R. J., \& Lee, J.-W. (2010). A new dataset of educational attainment in the world, 1950-2010 (Working Paper No. 15902). Cambridge, MA: National Bureau of Economic Research.

Basmann, R. L. (1957). A generalized classical method of linear estimation of coefficients in a structural equation. Econometrica, 25(1), 77-83. 
Becker, G. S. (1975). Human capital: A theoretical and empirical analysis, with special reference to education (2nd ed.). Chicago: University of Chicago Press.

Ben-Porath, Y. (1967). The production of human capital and the lifecycle of earnings. Journal of Political Economy, 75(4), 352-365.

Bhatti, S. H., Bourdon, J., \& Aslam, M. (2013). Economic returns to education in France: OLS and instrumental variable estimations. Lahore Journal of Economics, 18(2), 51-63.

Bound, J., Jaeger, D. A., \& Baker, R. M. (1995). Problems with instrumental variables estimation when the correlation between the instruments and the endogenous explanatory variable is weak. Journal of the American Statistical Association, 90(430), 443-450.

Butcher, K. F., \& Case, A. (1994). The effect of sibling sex composition on women's education and earnings. Quarterly Journal of Economics, 109(3), 531-563.

Card, D. (1993). Using geographic variation in college proximity to estimate the return to schooling (Working Paper No. 4483). Cambridge, MA: National Bureau of Economic Research.

Card, D. (1999). The causal effect of education on earnings. In O. C. Ashenfelter \& D. Card (eds.), Handbook of labor economics (vol. 3, pp. 1801-1863). Amsterdam: Elsevier.

Card, D. (2001). Estimating the return to schooling: Progress on some persistent econometric problems. Econometrica, 69(5), 1127-1160.

Chen, G., \& Hamori, S. (2009). Economic returns to schooling in urban China: OLS and the instrumental variables approach. China Economic Review, 20(2), 143-152.

Farooq, M., \& Sulaiman, D. J. (2009). Gender earnings inequality and discrimination in the Pakistani labor market. The Dialogue, 4(3), 373-385.

Griliches, Z. (1977). Estimating the returns to schooling: Some econometric problems. Econometrica, 45(1), 1-22. 
Griliches, Z. (1979). Sibling models and data in economics: Beginnings of a survey. Journal of Political Economy, 87(5, Part 2), S37-S64.

Gujarati, D. N. (2007). Basic econometrics (4th ed.). New Delhi: Tata McGraw-Hill.

Hausman, J. A. (1978). Specification tests in econometrics. Econometrica, $46(6), 1251-1271$.

Hyder, A. (2007). Wage differentials, rate of return to education, and occupational wage share in the labor market of Pakistan (Working Paper No. 17). Islamabad: Pakistan Institute of Development Economics.

Hyder, A., \& Reilly, B. (2005). The public and private sector pay gap in Pakistan: A quantile regression analysis. Pakistan Development Review, 44(3), 271-306.

Johnson, E. N., \& Chow, G. C. (1997). Rates of return to schooling in China. Pacific Economic Review, 2(2), 101-113.

Khan, F. S., \& Toor, I. A. (2003). Changes in returns to education in Pakistan: 1990-2002. Lahore Journal of Economics, 8(2), 83-98.

Kimmel, J. (1997). Rural wages and returns to education: Differences between whites, blacks, and American Indians. Economics of Education Review, 16(1), 81-96.

Korsun, V. (2010). Wage determination in Ukraine: Does religiosity matter? Unpublished Master's dissertation, Kyiv School of Economics, Kyiv.

Lassibille, G. (1998). Wage gaps between the public and private sectors in Spain. Economics of Education Review, 17(1), 83-92.

Mincer, J. (1958). Investment in human capital and personal income distribution. Journal of Political Economy, 66(4), 281-302.

Mincer, J. (1974). Schooling, experience and earnings. New York: Columbia University Press.

Qureshi, M. G. (2012). The gender differences in school enrolment and returns to education in Pakistan. Pakistan Development Review, 51(3), 219-256. 
Theil, H. (1953). Repeated least squares applied to complete equation systems. Unpublished manuscript, Central Planning Bureau, The Hague.

Willis, R. J. (1986). Wage determinants: A survey and reinterpretation of human capital earnings functions. In O. C. Ashenfelter \& R. Layard (eds.), Handbook of labor economics (vol. 1, pp. 525-602). Amsterdam: Elsevier.

Yasin, G., Chaudhry, I. S., \& Afzal, S. (2010). The determinants of gender wage discrimination in Pakistan: Econometric evidence from Punjab province. Asian Social Science, 6(11), 239-255. 


\section{Appendix}

Table A1: Correlation matrix, Punjab (male)

\begin{tabular}{lcccc}
\hline Variable & Lnwage & Education & Instrument 1 & Instrument 2 \\
\hline Lnwage & 1 & 0.3979 & 0.1408 & -0.2699 \\
& & $(<0.0001)$ & $(<0.0001)$ & $(<0.0001)$ \\
Education & 0.3979 & 1 & 0.515 & 0.4308 \\
& $(<0.0001)$ & & $(<0.0001)$ & $(<0.0001)$ \\
Instrument 1 & 0.1408 & 0.515 & 1 & -0.0542 \\
& $(<0.0001)$ & $(<0.0001)$ & & $(<0.0001)$ \\
Instrument 2 & -0.2699 & 0.4308 & -0.0542 & 1 \\
& $(<0.0001)$ & $(<0.0001)$ & $(<0.0001)$ & \\
\hline
\end{tabular}

Source: Authors' estimates.

Table A2: Correlation matrix, Punjab (female)

\begin{tabular}{lcccc}
\hline Variable & Lnwage & Education & Instrument 1 & Instrument 2 \\
\hline Lnwage & 1 & 0.393 & 0.1504 & -0.3321 \\
& & $(<0.0001)$ & $(0.001189)$ & $(<0.0001)$ \\
Education & 0.393 & 1 & 0.5398 & 0.449 \\
& $(<0.0001)$ & & $(<0.0001)$ & $(<0.0001)$ \\
Instrument 1 & 0.1504 & 0.5398 & 1 & -0.0142 \\
& $(0.001189)$ & $(<0.0001)$ & & $(0.760481)$ \\
Instrument 2 & -0.3321 & 0.449 & -0.0142 & 1 \\
& $(<0.0001)$ & $(<0.0001)$ & $(0.760481)$ & \\
\hline
\end{tabular}

Source: Authors' estimates.

Table A3: Correlation matrix, Sindh (male)

\begin{tabular}{lcccc}
\hline Variable & Lnwage & Education & Instrument 1 & Instrument 2 \\
\hline Lnwage & 1 & 0.4135 & 0.3036 & -0.3212 \\
& & $(<0.0001)$ & $(<0.0001)$ & $(<0.0001)$ \\
Education & 0.4135 & 1 & 0.4373 & 0.4758 \\
& $(<0.0001)$ & & $(<0.0001)$ & $(<0.0001)$ \\
Instrument 1 & 0.3036 & 0.4373 & 1 & -0.0253 \\
& $(<0.0001)$ & $(<0.0001)$ & & $(0.141475)$ \\
Instrument 2 & -0.3212 & 0.4758 & -0.0253 & 1 \\
& $(<0.0001)$ & $(<0.0001)$ & $(0.141475)$ & \\
\hline
\end{tabular}

Source: Authors' estimates. 
Table A4: Correlation matrix, Sindh (female)

\begin{tabular}{lcccc}
\hline Variable & Lnwage & Education & Instrument 1 & Instrument 2 \\
\hline Lnwage & 1 & 0.347 & 0.2396 & -0.2144 \\
& & $(<0.0001)$ & $(0.0001)$ & $(0.0005)$ \\
Education & 0.347 & 1 & 0.5197 & 0.3414 \\
& $(<0.0001)$ & & $(<0.0001)$ & $(<0.0001)$ \\
Instrument 1 & 0.2396 & 0.5197 & 1 & -0.1253 \\
& $(0.0001)$ & $(<0.0001)$ & & $(0.0448)$ \\
Instrument 2 & -0.2144 & 0.3414 & -0.1253 & 1 \\
& $(0.0005)$ & $(<0.0001)$ & $(0.0448)$ & \\
\hline
\end{tabular}

Source: Authors' estimates.

Table A5: Correlation matrix, KP (male)

\begin{tabular}{lcccc}
\hline Variable & Lnwage & Education & Instrument 1 & Instrument 2 \\
\hline Lnwage & 1 & 0.4739 & 0.104 & -0.3767 \\
& & $(<0.0001)$ & $(0.0001)$ & $(<0.0001)$ \\
Education & 0.4739 & 1 & 0.4393 & 0.5378 \\
& $(<0.0001)$ & & $(<0.0001)$ & $(<0.0001)$ \\
Instrument 1 & 0.104 & 0.4393 & 1 & -0.1136 \\
& $(<0.0001)$ & $(<0.0001)$ & & $(<0.0001)$ \\
Instrument 2 & -0.3767 & 0.5378 & -0.1136 & 1 \\
& $(0.0001)$ & $(<0.0001)$ & $(<0.0001)$ & \\
\hline
\end{tabular}

Source: Authors' estimates.

Table A6: Correlation matrix, KP (female)

\begin{tabular}{lcccc}
\hline Variable & Lnwage & Education & Instrument 1 & Instrument 2 \\
\hline Lnwage & 1 & 0.3524 & 0.1289 & -0.4564 \\
& & $(<0.0001)$ & $(0.1089)$ & $(<0.0001)$ \\
Education & 0.3524 & 1 & 0.3699 & 0.5059 \\
& $(<0.0001)$ & & $(<0.0001)$ & $(<0.0001)$ \\
Instrument 1 & 0.1289 & 0.3699 & 1 & -0.1005 \\
& $(0.1089)$ & $(<0.0001)$ & & $(0.2125)$ \\
Instrument 2 & -0.4564 & 0.5059 & -0.1005 & 1 \\
& $(<0.0001)$ & $(<0.0001)$ & $(0.2125)$ & \\
\hline
\end{tabular}

Source: Authors' estimates. 
Table A7: Correlation matrix, Balochistan (male)

\begin{tabular}{lcccc}
\hline \multicolumn{1}{c}{ Variable } & Lnwage & Education & Instrument 1 & Instrument 2 \\
\hline Lnwage & 1 & 0.4913 & 0.0723 & -0.3426 \\
& & $(<0.0001)$ & $(0.0088)$ & $(<0.0001)$ \\
Education & 0.4913 & 1 & 0.4365 & 0.5632 \\
& $(<0.0001)$ & & $(<0.0001)$ & $(<0.0001)$ \\
Instrument 1 & 0.0723 & 0.4365 & 1 & -0.0856 \\
& $(0.0088)$ & $(<0.0001)$ & & $(0.0019)$ \\
Instrument 2 & -0.3426 & 0.5632 & -0.0856 & 1 \\
& $(<0.0001)$ & $(<0.0001)$ & $(0.0019)$ & \\
\hline
\end{tabular}

Source: Authors' estimates.

Table A8: Correlation matrix, Balochistan (female)

\begin{tabular}{lcccc}
\hline \multicolumn{1}{c}{ Variable } & Lnwage & Education & Instrument 1 & Instrument 2 \\
\hline Lnwage & 1 & 0.5315 & 0.2008 & -0.394 \\
& & $(<0.0001)$ & $(0.0654)$ & $(0.0002)$ \\
Education & 0.5315 & 1 & 0.4127 & 0.5087 \\
& $(<0.0001)$ & & $(<0.0001)$ & $(<0.0001)$ \\
Instrument 1 & 0.2008 & 0.4127 & 1 & -0.1347 \\
& $(0.0654)$ & $(<0.0001)$ & & $(0.2192)$ \\
Instrument 2 & -0.394 & 0.5087 & -0.1347 & 1 \\
& $(0.0002)$ & $(<0.0001)$ & $(0.2192)$ & \\
\hline
\end{tabular}

Source: Authors' estimates. 